\title{
Inversión Reciente en Uruguay: ¿Hacia un Desarrollo Sostenible?
}

\author{
Cecilia Durán ${ }^{1}$, Alberto González ${ }^{2}$, Andrés Jung ${ }^{1^{*}}$, Silvia Vazquez ${ }^{1}$
}

Resumen: Este trabajo pretende aportar una primera aproximación sectorial al impacto de la inversión promovida en Uruguay entre 2010 y 2014 sobre el patrón de innovación y el mercado de trabajo. Desde una perspectiva de desarrollo sostenible e inclusivo, se concluye que la inversión promovida no se asocia con ningún patrón de innovación de las empresas a nivel sectorial, ni con variables que hacen al mercado laboral. Se identifican resultados primarios que muestran una asociación de los sectores con mayor inversión promovida en el período, con origen del capital, tamaño de las empresas, mayor productividad y promedio salarial del sector.

Palabras clave: inversión; promoción de inversiones; desarrollo sostenible; innovación

Abstract: This study intends to be a first sectorial approach to evaluate the impact of investment promotion in Uruguay between 2010 and 2014 , on firm innovation patterns and labor market variables. From a sustainable development perspective, no association is found between investments promoted by specific policies and labor market variables or firm innovation patterns at a sectorial level. The study finds some evidence of association between those industries with more promoted investment and the origin of capital, firm size, productivity and average wage at a sectorial level.

Keywords: investment; investment promotion policy; sustainable development; innovation

$21^{\text {st }}$ International Association of Jesuit Business Schools (IAJBS)

$18^{\text {th }}$ Colleagues in Jesuit Business Education (CJBE)

\section{Motivación}

En Durán et al. (2012) se identificaron algunos aspectos clave de política económica que podían incidir en la generación de capacidades productivas para sostener un desarrollo armónico de futuro, basado en criterios de equidad e igualdad de oportunidades. Esta reflexión iba en la línea de diversos autores que vinculan el desarrollo de los países a los procesos de diversificación productiva (Hausmann \& Rodrik, 2003; Imbs \& Wacziarg, 2003). En una economía pequeña, por su parte, la estructura productiva tiene mucho que ver con la estructura de exportaciones (Hausmann et al., 2007; Vázquez et al., 2011).

Uruguay ha registrado en los últimos años un fuerte dinamismo de las exportaciones, de la economía en general y del sector manufacturero en particular, que ha sido acompañado por una serie de cambios estructurales, aunque asociado a un proceso de concentración de las ventas externas en pocos productos. Si bien la estructura de las exportaciones se ha diversificado por destinos, se ha concentrado en productos de base agropecuaria con escaso valor agregado manufacturero (Vázquez et al., 2011).

Si bien es cierto que no todo el valor agregado se genera en la fase manufacturera, que los servicios tienen una importancia creciente, incluso asociados a commodities, y que cada vez más se innova a nivel agropecuario, este perfil empresarial, por productos y por nivel tecnológico, alimenta algunas interrogantes. Estas se vinculan con la estructura productiva, la concentración de exportaciones y el papel de la inversión como generadora de crecimiento con diversificación productiva y generación de capacidades para el desarrollo
(Jung \& Vázquez, 2013, Plottier et al., 2013). Estrategias y decisiones de inversión que diversifiquen la matriz productiva y generen capacidades y empleo de calidad, contribuirán al desarrollo sostenible y la equidad social (Durán et al., 2012; Garbarino et al., 2008).

En Uruguay, la tasa de inversión privada pasó de 11,1\% en 2004 a $18,3 \%$ del PIB en 2013. Este boom de la inversión, y en particular de la IED que se multiplicó por 9 en diez años, es resultado de shocks externos positivos (como el dinamismo en los precios internacionales de productos primarios y la fuerte liquidez a nivel global), pero también del uso activo de instrumentos de política y de decisiones de actores empresariales locales e internacionales.

Los hechos más novedosos en materia de inversión en los últimos años están asociados a los grandes proyectos de producción de celulosa y, más recientemente, a la producción de energía -principalmente eólica. En lo restante, la estructura de inversiones promovidas ha tendido a profundizar la concentración ya existente en el sector de Alimentos y bebidas, a la vez que no da señales de recuperación de sectores altamente intensivos en mano de obra que se han venido contrayendo en los últimos años (Vázquez \& Jung, 2014).

Con el objetivo de avanzar en la mejor comprensión de los cambios en la estructura productiva y de exportaciones y en las perspectivas de un desarrollo sustentable, en un contexto de cambio de ciclo económico para los países de la región, el presente estudio pretende analizar el patrón de inversiones promovidas que se ha registrado en los últimos años (2010-2014), para luego evaluar los posibles impactos que el mismo puede tener en términos de la estructura productiva

(1) Departamento de Economía, Facultad de Ciencias Empresariales, Universidad Católica del Uruguay.

(2) Departamento de Ciencias de la Administración, Facultad de Ciencias Empresariales, Universidad Católica del Uruguay

* Corresponding author: ajung@ucu.edu.uy

ISSN: 0718-2724. (http://jotmi.org)

Journal of Technology Management \& Innovation @ Universidad Alberto Hurtado, Facultad de Economía y Negocios.

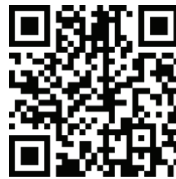

102 
sectorial. Considerando este trabajo como una primera exploración de tales impactos, en las secciones que siguen se describe brevemente la evolución de la inversión en Uruguay en los últimos años y el marco normativo que la promociona (sección 2). Luego de explicitar la aproximación metodológica de este trabajo (sección 3), se analizan las características de la inversión promovida (sección 4), y de los sectores a que se asigna (sección 5). En la sección 6 se intenta identificar la asociación de la inversión promovida con el patrón sectorial de la estructura productiva, en particular atendiendo al perfil sectorial de la innovación y empleo. Finalmente se presentan algunas reflexiones que surgen del análisis realizado.

\section{Antecedentes}

Hacia 2003 la economía uruguaya emprendía la salida de la profunda crisis que había tenido su mayor impacto en el año 2002. Tras la crisis se inició una senda de dinámico crecimiento, que alcanzó un promedio anual de 6,2\% entre 2005 y 2010. No obstante, desde 2012 el crecimiento comenzó a desacelerarse, convergiendo a un ritmo más en línea con el histórico (en el entorno de 3\% anual).

\subsection{Evolución de la inversión privada en los últimos 10 años}

La inversión ha mostrado un muy importante dinamismo a partir de 2006, que en el caso de la IED muestra características de boom.

La tasa de inversión de la economía creció de forma importante, pasando de 12,3\% en 2003 a 21,4\% en 2014, y esto en el marco de un PIB muy dinámico. A su interior, la tasa de inversión privada pasó de $9,3 \%$ en 2003 a 16,8\% en 2014, alcanzando registros de 18,3\% en 2012. Pese a ello, dada la estructuralmente baja tasa de inversión de la economía, aún permanece por debajo del promedio de América Latina.

\section{Gráfico 1: Dinamismo de la economía y de la inversión e IED}

Tasa de variación a precios constantes (\%) y miles de millones de USD

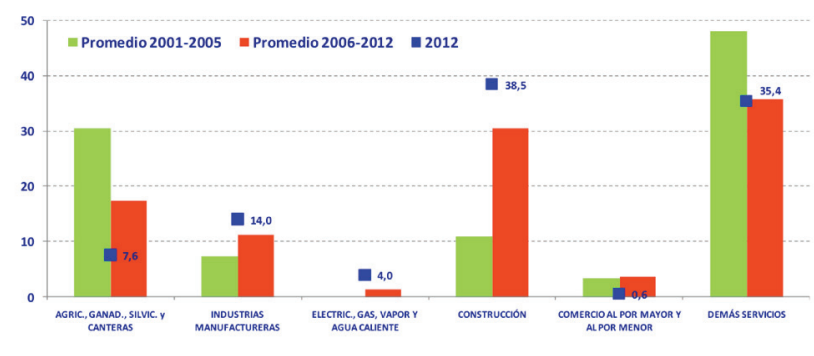

El fuerte dinamismo de la inversión privada sería producto de la interacción de diferentes factores. Se profundizó una política activa de promoción de inversiones (Vázquez, 2014), en el marco de una larga fase expansiva del ciclo económico que operó como un "efecto acelerador", sumado a factores externos (abundante liquidez internacional, menor costo del financiamiento, fuertes flujos de IED en busca de rentabilidad), y a un ambiente de negocios confiable.
La inversión pública, por su parte, que representa en general un $20 \%$ del total, está focalizada en infraestructura si se excluye la inversión de las empresas públicas (el $81,3 \%$ de la inversión pública en el promedio de 2005-2014 es bajo la modalidad de construcción).

\subsection{Evolución y estructura de la Inversión Extranjera Directa}

Los flujos de IED netos hacia Uruguay crecieron más que proporcionalmente que los orientados a América Latina y al Mercosur. Pasaron de US\$ 416,4 millones en 2003 a US\$ 3.030,3 millones en 2013.

Si bien todos los sectores observan un importante aumento de IED, el diferente ritmo de crecimiento pautó un cambio en su estructura sectorial.

Considerando la IED acumulada entre 2001 a 2005 y 2006 a 2012, según surge de la clasificación del BCU, la construcción sería el sector cuya participación más creció, pasando de captar en promedio el $11,0 \%$ al 30,5\% del total. Cabe señalar que en el año 2006 comenzó la ejecución de la instalación de la planta procesadora de pasta de celulosa UPM (entonces Botnia), la mayor IED hasta ese momento en la historia del país, marcando un punto de inflexión en el ritmo de ingreso de IED a esta economía. En segundo lugar como sector "ganador" está la industria manufacturera, que pasó de captar el 7,4\% al 11,2\% del total. En la misma línea se aprecia la participación del sector de electricidad, gas y agua, en un proceso de diversificación de la matriz energética del país, que alcanzó a 4,0\% en 2012.

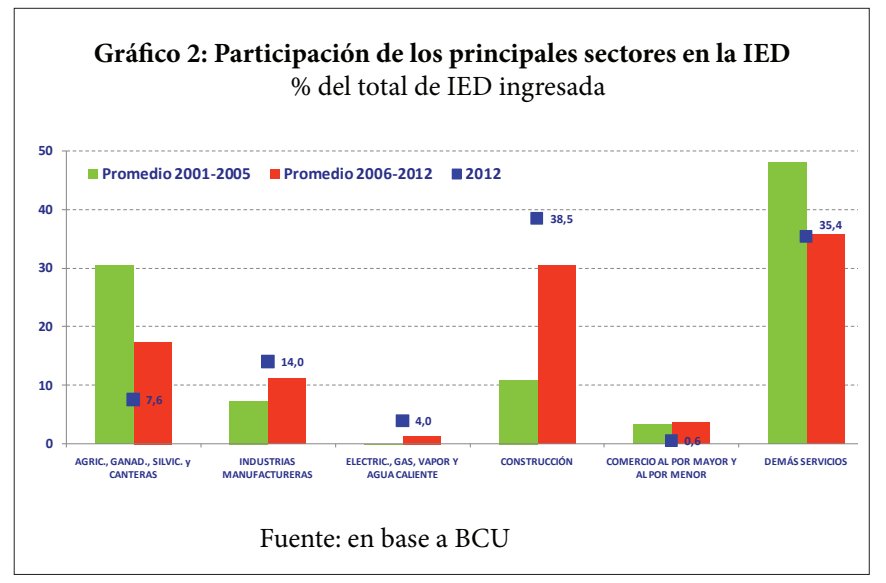

Entre los sectores que, pese a absorber un mayor monto en dólares, perdieron participación en la captación en los últimos años, está el sector primario -que agrupa agricultura, ganadería, caza, pesca y canteras, pasando de $30,5 \%$ a $17,5 \%$ del total, seguido del sector servicios -excepto comercio- que pasó de $48,1 \%$ a 35,1\%. Hacia 2012 se continuaban profundizando esas pérdidas de participación.

\section{Mecanismos de promoción de inversión}

En Uruguay, asignar importancia a la inversión privada, constituye una política de Estado desde hace décadas. En tal sentido, los primeros avances datan dela década del ' 70 , aunque el régimen vigentey relevante para este período de estudio, sería el regulado por el decreto del año 2007, con ajustes provenientes del decreto del año 2012. 
Tal como se la ha entendido, la política de promoción de la inversión trasciende los meros incentivos fiscales, en la medida que además requiere de un contexto de estabilidad socio-económica, y en particular, político-institucional, donde resultan críticas las reglas de juego. Entre ellas, por ejemplo, la libre repatriación de capital y utilidades y la no discriminación entre inversión nacional y extranjera.

Los principales instrumentos directos vigentes para la promoción de inversiones en el país son:

- Ley de promoción y protección de inversiones. Esta ley, cuya primera versión data de 1974, se aplica de forma horizontal, y brinda igual trato a inversores nacionales y extranjeros, garantizando principios básicos como la libre repatriación de utilidades. Se otorgan importantes beneficios fiscales, que pueden llegar a la exoneración del impuesto a la renta por un $100 \%$ del monto invertido, dentro de otros beneficios impositivos. El nivel de este beneficio está condicionado al cumplimiento por parte de los proyectos de una serie de indicadores, que toman en cuenta, dentro de otras cosas, el nivel y calidad del empleo y el gasto en innovación.

- $\quad$ Ley de Zonas Francas. Uruguay cuenta con un régimen de zonas francas que exonera a las empresas de la totalidad de los impuestos, con excepción de la seguridad social, de forma permanente, y que opera tanto para el sector industrial, comercial y de servicios. En los últimos años el régimen se ha expandido fuertemente, aprobándose el beneficio para UPM y Punta PereiraMontes del Plata (ambos grandes emprendimientos industriales específicos), Aguada Park y WTC (Servicios globales) y Parque de las Ciencias (Servicios especializados).

- Regímenes sectoriales específicos. Varios sectores en Uruguay cuentan con regímenes específicos para promover las inversiones, tanto en el marco de la Ley de Inversiones como al margen de ésta. Sector naval, electrónica, biotecnología, vivienda, forestal, software, son solo algunos ejemplos.

- Parques industriales, puertos y aeropuertos libres, contratos de participación público privada, junto con una variada oferta de regímenes vinculados al comercio exterior, complementan el régimen de atracción de inversiones de Uruguay.

Asimismo, esta promoción se enmarca en un sistema de política industrial activa que fue creando una serie de instrumentos focalizados en determinados objetivos y sectores, que contribuyen al mismo fin de desarrollo, e indirectamente estimulan la inversión.

Estudios recientes constatan resultados positivos de la política en mayor inversión, creación de empleo, especialmente en empresas más intensivas, y acceso de PYMEs a los beneficios (Gervaz, 2012; Peláez, 2012; Carbajal et al., 2014; Llambí \& Cazulo, 2014).

\section{Aproximación metodológica}

Dado el objetivo planteado para este trabajo se utilizaron bases de datos de proyectos de inversión promovidos (PIP) bajo los instrumentos de política diseñados para este fin. Esta es la única información disponible que permite un abordaje sectorial para el análisis de la inversión.

La información sobre inversión promovida surge de los datos sobre proyectos aprobados por COMAP (Ministerio de Economía y Finanzas, MEF). Se agregaron los PIP a nivel de empresa en el total del período 2010-2014. La información es presentada en dólares e identifica, según cuál sea el ministerio evaluador, el sector al que pertenece: agro, comercio, industria, turismo y servicios. Se procedió a clasificar los datos según actividad principal de la empresa, en CIIU revisión 4, a dos dígitos. Se entiende este es uno de los aportes de este trabajo, en la medida que permitirá a futuro realizar más estudios a nivel de empresa teniendo clasificado su sector de actividad.

La información de proyectos promovidos por COMAP fue complementada con la proveniente de proyectos ejecutados bajo el régimen de Zona Franca. En la medida que la inversión promovida desde COMAP se ha ejecutado en los primeros años en un porcentaje mayor al 80\% (Llambí \& Cazulo, 2014), se agregó la información de ambas fuentes al interior del período.

A efectos de caracterizar los sectores -CIIU 2 dígitos- se utilizó información proveniente de dos fuentes: Instituto Nacional de Estadísticas (INE) y Agencia Nacional de Investigación e Innovación (ANII).

La información del INE utilizada es la Encuesta Anual de Actividad Económica (EAAE) para 2008-2011, que abarca empresas de la industria manufacturera y la mayor parte de servicios. Queda excluida la información de los sectores primarios, de construcción, actividades inmobiliarias y sector financiero. Los datos de ANII corresponden a la Encuesta de Innovación en Industria y Servicios 2010-2012 (ANII, 2014). La información cubre los sectores de la industria manufacturera y del sector servicios con la excepción de comercio, sistema financiero y otros de menor contribución. La cobertura sectorial de las bases del INE y de la ANII, imponen una cierta limitación al estudio, que debe tenerse en cuenta al considerar las conclusiones del mismo.

En cuanto al análisis, se realiza una primera etapa descriptiva a efectos de "mapear" las características de los sectores que invirtieron al amparo de los mecanismos de promoción.

Al realizar un primer abordaje sectorial, se trata de identificar correlaciones (gráficos de ploteo con R2) entre variables que hacen a los montos de inversión promovida, con variables que caracterizan a los sectores. Esto se realiza en dos etapas: a nivel de "letra" (12 grandes sectores de actividad) y a nivel de 2 dígitos CIIU rev 4 (67 sectores para variables INE y en 47 sectores para variables ANII). Las medias sectoriales en el caso ANII excluyen empresas públicas. 
En una segunda etapa, se aborda el análisis a través de herramientas específicas de análisis estadístico, utilizando el Análisis de Componentes Principales (ACP). A través del ACP se busca identificar perfiles de innovación entre los sectores y evaluar la existencia o no de correlaciones con las variables asociadas a los montos de inversión promovidos a nivel sectorial. Este análisis busca identificar si la política de promoción de la inversión está logrando impactar positivamente en variables claves para avanzar hacia un modelo de desarrollo sostenible e inclusivo.

\section{La inversión promovida en Uruguay 2010-2014}

\subsection{La importancia de la Inversión promovida}

Considerando el período 2010-2014, los proyectos promovidos en el marco del Régimen de promoción de inversiones alcanzaron los USD 9.393 millones recomendados por COMAP y USD 2.574 millones promovidos por el régimen de Zonas Francas, lo que implica un total de USD 11.967 millones

La Ley de promoción de inversión y el régimen de Zonas Francas son muy abarcativos en materia sectorial, y desde la reglamentación de 2007 prácticamente sólo quedarían excluidas la inversión en inmuebles destinados a vivienda, y algunos tipos de activos (por ejemplo, automotores no utilitarios).

Si se considera la clasificación institucional de la formación bruta de capital fijo, la inversión privada históricamente ha contribuido en el entorno del $80 \%$ (78,6\% en el promedio de los últimos 10 años). Al interior de la inversión privada, para el promedio de 2010-2014, el $57,1 \%$ fue en construcción. Si se considera que desde 2007 la Ley de Promoción de Inversiones abarca todos los sectores de actividad excepto la inversión para vivienda, se podría estimar que el universo de inversión potencialmente beneficiaria de este régimen de promoción sería toda la inversión privada menos "Edificios" (cuya construcción se asume privada ante la imposibilidad de identificar su origen a partir de la información disponible en BCU) y "activos cultivados". Esto dejaría la inversión privada acumulada entre 2010 y 2014, potencialmente beneficiaria de los regímenes de promoción, en USD 18.100 millones.

Dados los montos promovidos por los regímenes considerados, entre ambos instrumentos se promovió el equivalente al 66\% de la inversión que podría acceder a los beneficios tributarios previstos por ellos.

Por otra parte, según surge del informe de la UNASEP (2014), a partir de un estudio de seguimiento que se realiza de la inversión promovida entre 2008 y 2013, se ejecutó el $81 \%$ de la inversión comprometida. En particular, en el sector de industria manufacturera este cumplimiento alcanzó al $96 \%$, en el comercio fue $83 \%$, en transporte $86 \%$, servicios sociales y salud $95 \%$, por citar algunos de los más relevantes.

Por su parte, el monto de inversiones que ingresaron entre 2010 y 2014 bajo el régimen de Zona Franca presenta una elevada concentración en una IED con destino a la producción de pulpa de celulosa (Montes del Plata: USD 2.075 millones). De los USD 500 millones restantes,
USD 95 millones tenían como destino el Parque de las Ciencias, y los restantes USD 400 millones se distribuyeron en otros servicios. Estos proyectos también fueron ejecutados básicamente por la totalidad de los montos comprometidos.

En este marco, se puede considerar que, analizando los proyectos promovidos bajo ambos regímenes, se puede contar con una panorámica razonablemente representativa de la inversión que se ha ejecutado en el país en el período de referencia. Esta información permite tener una aproximación sectorial actualizada -única disponible al análisis de la formación bruta de capital fijo de los diferentes sectores, de modo a poder inferir desde esa distribución de la inversión, los efectos sobre el patrón de desarrollo productivo de los años venideros.
Gráfico 3: Participación de la inversión promovida por los
regímenes de promoción

Porcentaje de la inversión elegible, 2010 - 2014

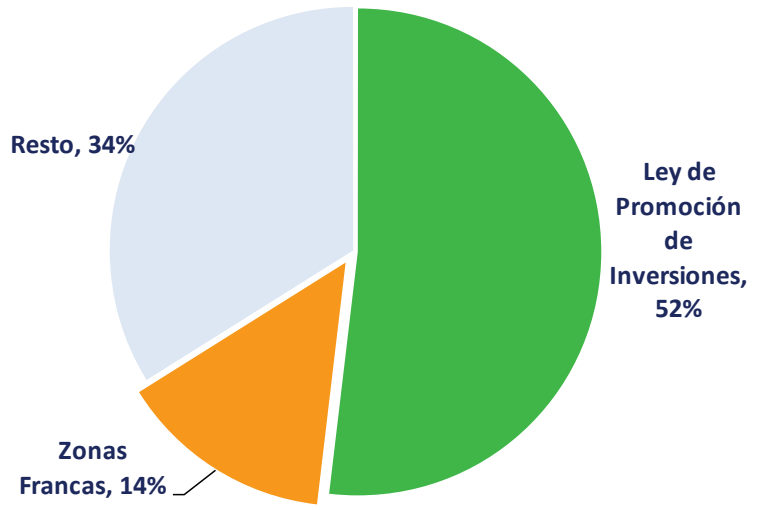

Fuente: Elaboración propia en base a BCU - MEF - COMAP
4.2 Principales características de la inversión promovida 2010 - 2014

Los proyectos promovidos por la Ley de Inversiones en el período 2010-2014 profundizan lo que se observa en el patrón de la formación bruta de capital fijo (FBKF) que se ejecutó en el período previo, con una fuerte participación de la electricidad vinculada a la apuesta al cambio de matriz energética realizada por el país. Excluyendo este rubro, la participación de la industria en los montos de inversión promovida es mayor a la que observa en la FBKF previa y en el PIB. Esta situación se amplifica si se consideran las inversiones en zona franca, en la medida que, de acuerdo con los últimos datos disponibles, las empresas manufactureras representaban únicamente el $2 \%$ de los establecimientos en 2010, pero explicaban el $45 \%$ del VAB, y esto sin considerar que la principal inversión del rubro no estaba operativa en dicha fecha.

En lo que respecta a las inversiones en zona franca, la información disponible es limitada, -el último censo fue en 2010- por lo que se trabaja con valores a nivel sectorial estimados partir de información secundaria. En el período 2010-2013 se verifica la mayor inversión 
privada extranjera del país (Montes del Plata), que explica el alto peso del sector industrial en el período. En el 2009 se autorizó la instalación de una nueva zona franca, Parque de las Ciencias, con especialización en el sector farmacéutico. Dentro del sector servicios sigue predominando trading, logística, servicios profesionales y financieros.

En lo que respecta a las inversiones promovidas en el marco de la Ley de Inversiones, entre los años 2010 y 2014 se recomendaron 3.642 proyectos de inversión que corresponden a 2433 empresas, lo que evidencia que varias empresas utilizan de forma recurrente el instrumento, aun en un período tan breve como el analizado. Los proyectos de las principales 10 empresas acumularon el 18\% del total invertido en el período, mostrando una alta concentración.

Los proyectos recomendados por la COMAP mostraron un incremento continuo en valores entre 2010 y 2013. El año 2014, no obstante, muestra un quiebre con una caída del orden de $48 \%$ en valores. Esta caída se explica por una muy fuerte caída en los sectores comercio e industrial, que no llega a compensarse por el crecimiento mostrado por el sector agropecuario y de servicios.

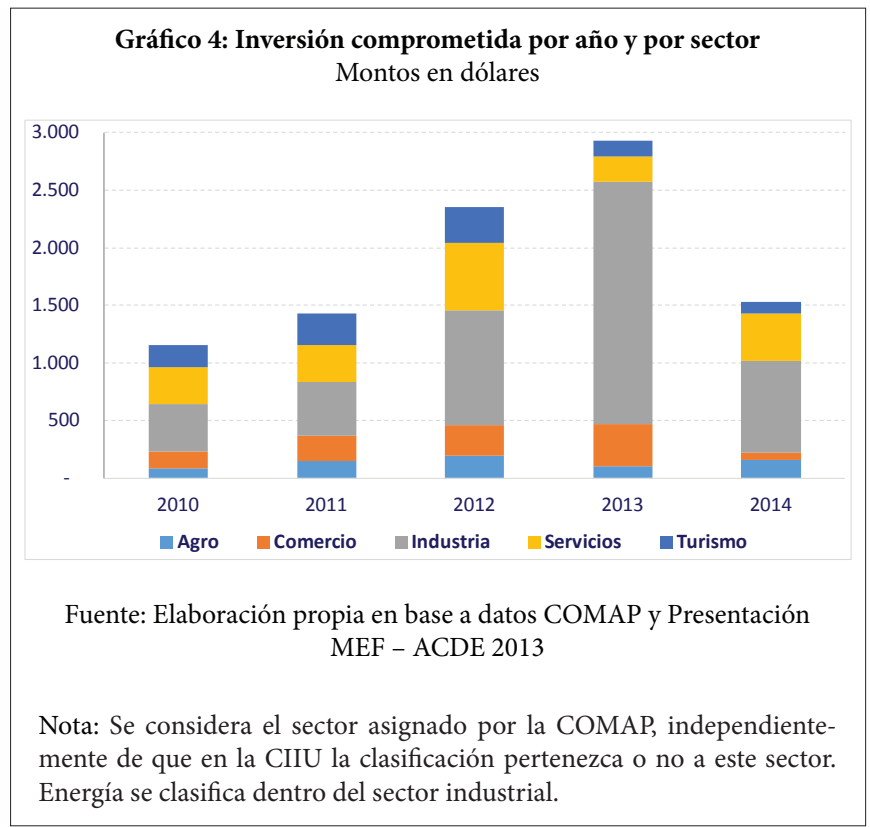

De acuerdo con la clasificación COMAP, un 51\% del valor comprometido en el período se corresponde al sector industrial, seguido del sector servicios con un $20 \%$. El turismo y el comercio representan un $11 \%$ cada uno y el agro un $7 \%$.

La industria por lo tanto explica la mayor parte de la inversión comprometida del período, lo que no se refleja en términos de número de proyectos promovidos, donde comercio y servicios tienen una participación superior (Gráfico 5). Esto es resultado de proyectos de inversión más grandes en el sector industrial. Estos tienen un valor promedio en el periodo que es cinco veces superior a los proyectos comerciales y tres veces a los de servicios.
La proporción sectorial en términos de empleo comprometido es similar a la participación en términos de cantidad de proyectos. Los sectores más intensivos en empleo son comercio y servicio, con una participación del 29\% cada uno en el período considerado, seguidos por el sector industrial. La inversión por empleo generado es seis veces mayor en la industria respecto al comercio y tres veces más que los servicios y el turismo.

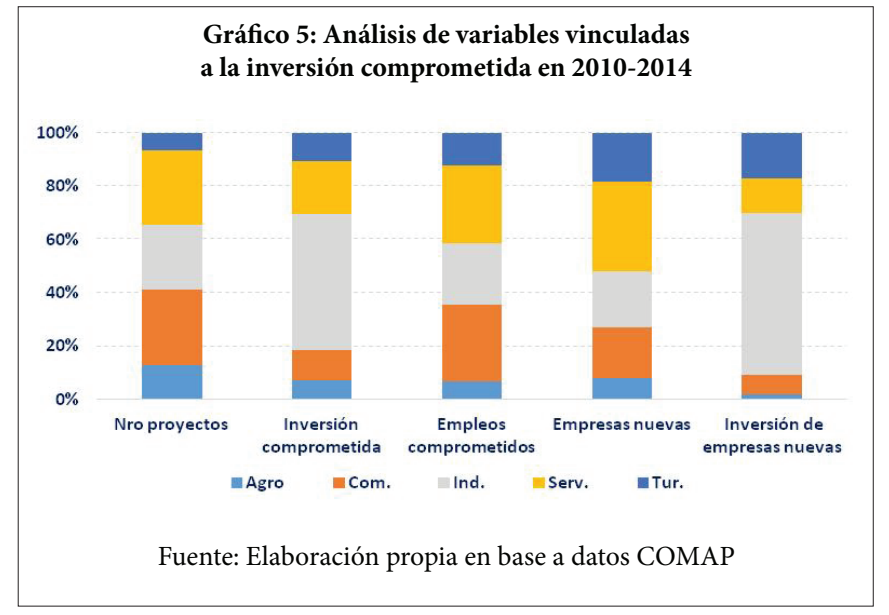

Los beneficios de la Ley de Inversiones aplican tanto para empresas nuevas como en marcha. En el período, un $17 \%$ de los proyectos de inversión se correspondieron a empresas nuevas. De estas, la mayor participación en términos de empresas generadas es para los servicios (34\%) seguido por la industria (21\%).

Las empresas nuevas representaron el 45\% de la inversión del período, confirmando una hipótesis esperable de que nuevos proyectos implican montos de inversión más altos que los correspondientes a empresas en marcha. Si bien en todos los sectores la participación de las empresas nuevas es mayor en valor que en cantidad, esta diferencia es mayor en industria y turismo, en ese orden, situación esperable dados los montos de inversión inicial asociados a estos sectores. Si se analiza la participación sectorial de la inversión por parte de empresas nuevas, el mayor porcentaje se corresponde al sector industrial, con un $61 \%$ del total, dominado por los nuevos proyectos en la generación de energía eólica, que se ubican entre los proyectos de mayor volumen del período.

\section{Impacto de los PIP por grandes sectores de actividad de la economía nacional}

La inversión promovida del período está concentrada en algunos rubros y sectores, tal como se desprende de la sección anterior. Interesa analizar qué características tienen esos sectores en términos de variables de relevancia, como ser valor agregado, empleo e innovación, y el vínculo que pueda haber con la inversión. En esta sección se realiza un análisis de orden cualitativo, intentando una primera aproximación empírica en la sección 6. 


\section{1- Análisis a nivel de rama de actividad}

Si bien un análisis univariante siempre aporta una visión parcial, interesa conocer cuál es el vínculo observado en el período bajo análisis de la inversión respecto al VAB, el capital fijo, el empleo y el salario.

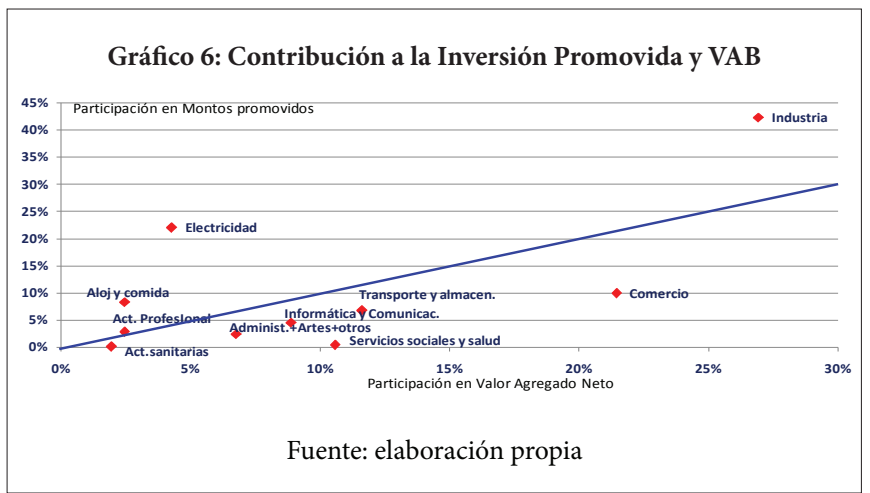

La inversión promovida muestra una relación ligeramente positiva con la contribución al porcentaje de valor agregado bruto (neto de impuestos y subsidios), siendo la industria el sector que más participación acumula en ambas variables, tanto si incluye o no el sector de la celulosa en el análisis. Considerando los tres principales sectores en términos de inversión, industria y electricidad muestran un nivel de inversión por encima del promedio en relación con su nivel de VAB, y comercio una participación por debajo.

En términos de contribución al stock de capital fijo también se observa una relación positiva con el monto invertido, mostrando que invierten más los más intensivos en capital, resultado esperable. Si bien la industria está invirtiendo por encima de lo que sería la tasa promedio, este valor se nivela si se excluye a la celulosa. Transporte y almacenamiento e informática y salud, por otra parte, estarían mostrando una tasa de inversión más baja de lo esperable para su intensidad de capital. En el caso de servicios sociales y de salud, los mecanismos analizados no son los más adecuados para captar la inversión generada por lo que el resultado no es significativo.

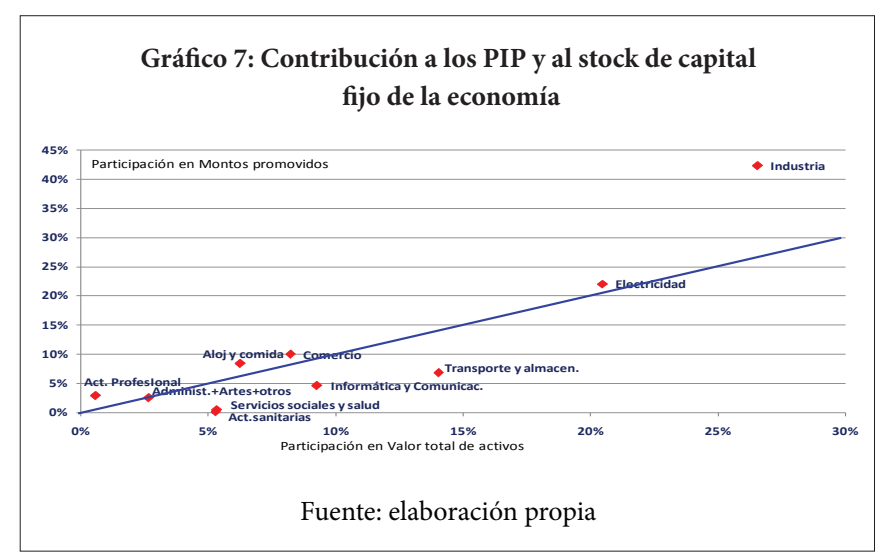

En términos de puestos de trabajo ocupados, si bien existe una relación positiva entre monto invertido y personal empleado, la dispersión es mayor. Electricidad es uno de los sectores que mayor inversión realizó en el período y menos contribuye al empleo.

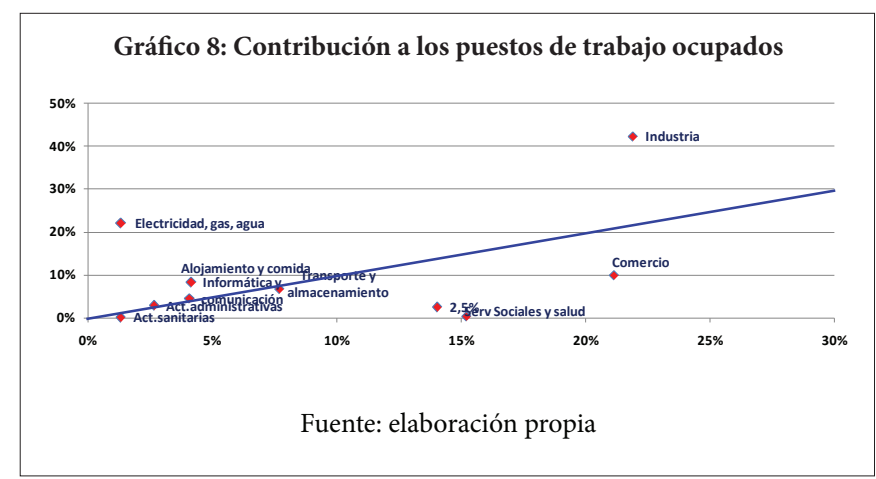

Exceptuando Electricidad gas y agua, cuya estructura de empleo está dominada por empresas públicas, los sectores que acumulan mayor participación en la nueva inversión -industria, comercio y alojamiento, comidas- no son los que más contribuyen a empleo de calidad en cuanto promedio salarial del sector.

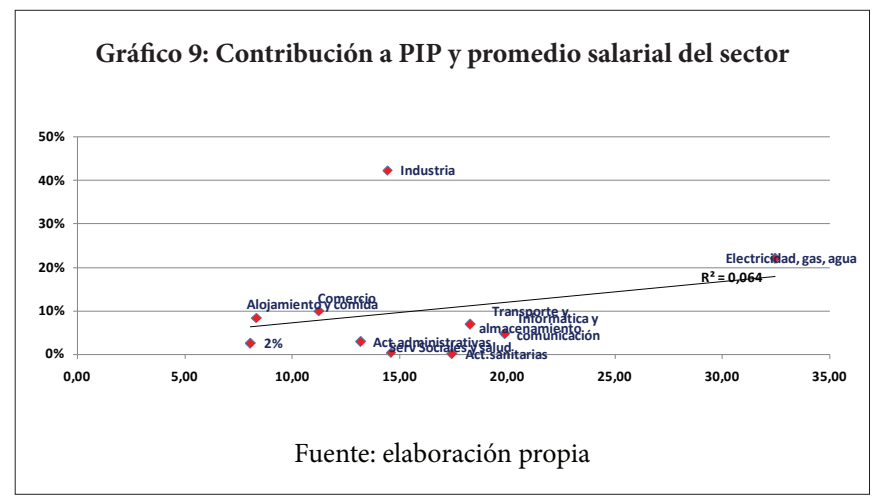




\section{2- Análisis por sector CIIU a dos dígitos}

El Cuadro 1 presenta los principales sectores y rubros que acumulan la mayor participación en la inversión promovida del período. La industria manufacturera representa el principal rubro, con dos sectores explicando casi el $75 \%$ de su participación, la industria papelera (47\%) y la industria alimenticia (23\%). Servicios y energía son los rubros que siguen en términos de participación, destacando las actividades de alojamiento en el primer caso que explican el 26\% del rubro.

Cuadro 1 - Principales sectores con PIP COMAP y Zona Franca Participación porcentual en monto total. 2010-2014

\begin{tabular}{|c|c|c|}
\hline Rubro & Sector & $\%$ \\
\hline \multirow{2}{*}{$\begin{array}{l}\text { Agropecuaria, } \\
\text { Forestación y Pesca }\end{array}$} & Producción agropecuaria & $3,2 \%$ \\
\hline & Otros & $0,6 \%$ \\
\hline \multirow[t]{6}{*}{ Ind. Manufacturera } & Pasta de celulosa, papel y productos & $17,6 \%$ \\
\hline & Alimentos & $8,5 \%$ \\
\hline & Otros productos minerales no metálicos & $2,3 \%$ \\
\hline & Sustancias y productos químicos & $2,2 \%$ \\
\hline & Productos farmacéuticos & $2,0 \%$ \\
\hline & Otros & $4,5 \%$ \\
\hline Energía & Energía & $19,3 \%$ \\
\hline \multirow[t]{2}{*}{ Comercio } & Comercio por mayor y menor (exc. Automotriz) & $8,2 \%$ \\
\hline & Otros & $0,5 \%$ \\
\hline \multirow[t]{6}{*}{ Servicios } & Alojamiento & $7,0 \%$ \\
\hline & Actividades inmobiliarias & $4,8 \%$ \\
\hline & Telecomunicaciones & $3,3 \%$ \\
\hline & Depósito y actividades complementarias & $3,1 \%$ \\
\hline & Transporte por vía terrestre & $1,5 \%$ \\
\hline & Otros & $7,2 \%$ \\
\hline Otros & & $4,2 \%$ \\
\hline \multicolumn{3}{|c|}{$\begin{array}{c}\text { Fuente: Elaboración propia en base a datos COMAP } \\
\text { y Presentación MEF - ACDE } 2013\end{array}$} \\
\hline \multicolumn{3}{|c|}{$\begin{array}{l}\text { Nota: La inversión total en zona franca se obtuvo de MEF (2013). La asig- } \\
\text { nación sectorial se realizó en base a cifras de Vaillant \& Lalanne (2014) } \\
\text { En los casos donde esta asignación no permitía una desagregación com- } \\
\text { parable con la utilizada para los proyectos de la COMAP, se hizo una } \\
\text { asignación en función del peso de cada CIIU } 2 \text { dígitos en estos proyectos. }\end{array}$} \\
\hline
\end{tabular}

Si se realiza el análisis por sector, independientemente del rubro, los doce sectores que cuentan con una inversión superior a la media acumulan el $81 \%$ de la inversión en el período (Gráfico 10). Se realiza un análisis diferenciado de los tres grupos identificados.

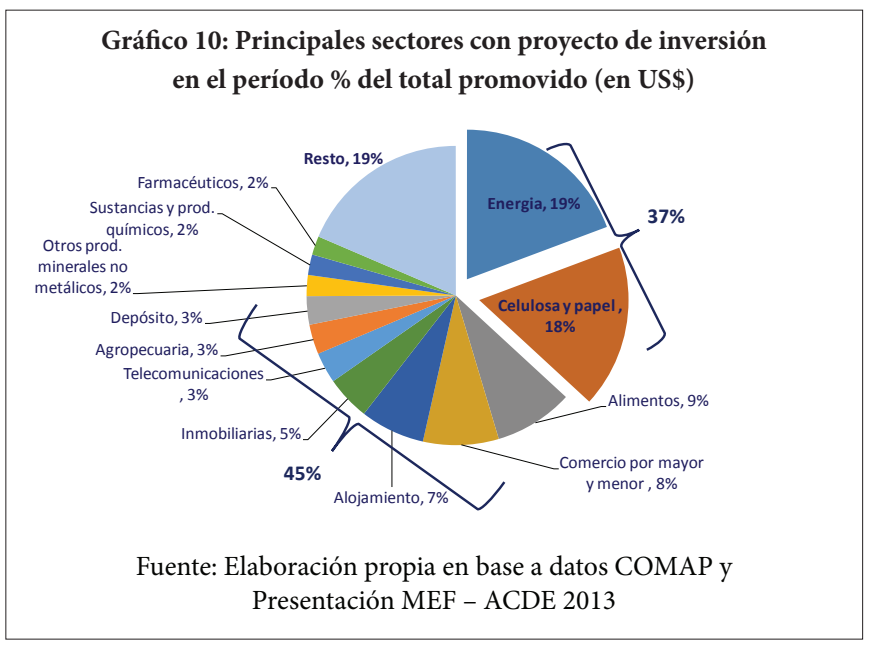

\subsection{1- Energía y celulosa}

Los dos principales sectores, energía y pasta de celulosa, representan en conjunto el $37 \%$ de la inversión analizada en el período siendo a su vez los dos principales exponentes de cada uno de los regímenes considerados. Se trata de inversiones concentradas en pocos proyectos y empresas, lo que es esperable, dado que la escala en el sector es relevante y el volumen de inversión por proyecto es significativo.

En términos de empleo, el sector de celulosa emplea por empresa más que el promedio, no así el de electricidad, situación que se invierte en términos de participación de profesionales y técnicos. Los dos sectores tienen valor agregado y remuneraciones por personal ocupado por encima de la media sectorial. En lo que hace a indicadores de innovación, la proporción de I+D sobre el total de ventas por empresa es menor a la media sectorial, identificándose en el caso de la celulosa una participación mayor al promedio en el caso de innovación a nivel de empresa en productos y procesos. En ambos casos, se tratan de capitales de origen extranjero.

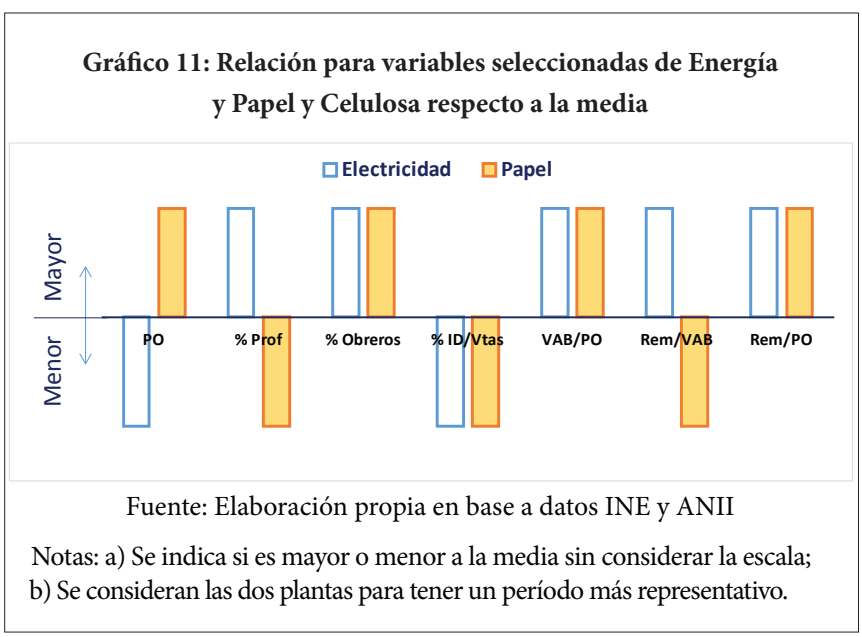

\subsection{2- Resto de sectores con inversión superior a la media}

Excluyendo energía y celulosa, los diez sectores con un monto de inversión promovida superior al promedio en el período, acumulan el $45 \%$ de la inversión y el 59\% de los proyectos. La participación sectorial es diversa, con presencia del sector agropecuario, industria (alimentos, química, farmacéuticos), comercio y servicios (alojamiento, depósito, telecomunicaciones) (Gráfico 10). Estos diez sectores representan aproximadamente el $45 \%$ del VAB y VBP del período y un $37 \%$ del empleo (excluido el sector agropecuario).

Los sectores analizados presentan una estructura de empresas privadas de menor porte que el promedio de la economía y con menor proporción de profesionales. También se observa una inversión en I + D inferior a la media en todos los sectores salvo farmacéutico (excluyendo para la media el valor correspondiente a la actividad de "investigación y desarrollo" -CIIU 72). El sector farmacéutico se destaca del resto en el sentido de ser el único que presenta valores por encima de la media en todas las variables analizadas menos una. 
En términos de remuneraciones, todos los sectores analizados presentan un valor inferior a la media en términos de participación en el VAB, siendo en comportamiento variable en materia de remuneración por trabajador. Depósito, telecomunicaciones, fabricación de minerales no metálicos, químicos y farmacéutico son quienes muestran una remuneración por encima del promedio.

Gráfico 12: Relación respecto a la media para variables seleccionadas

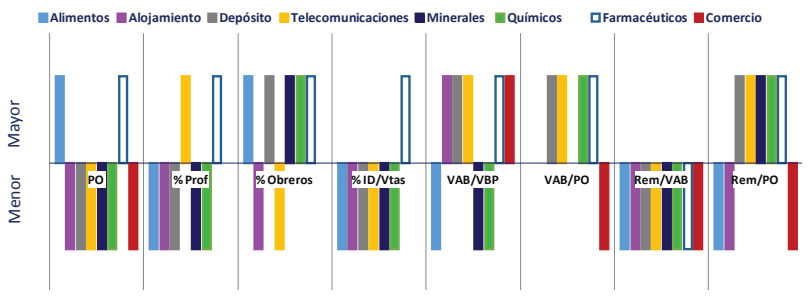

Fuente: Elaboración propia en base a datos INE y ANII

Nota: Se incluyen los sectores para los que se tenía información correspondiente a las variables relevadas con fuente ANII e INE.

\subsection{3- Resto de los sectores}

Los más de cincuenta sectores restantes, acumulan el 17\% del total de inversión promovida en el período y un $37 \%$ de la cantidad de proyectos, dando cuenta de la presencia de proyectos de menor tamaño.

El gráfico 11 analiza cómo se posicionan los sectores respecto a la media en una serie de variables de interés, y que porcentaje se encuentra mejor posicionado que el total. De acuerdo con lo que se observa, estos sectores contribuyen en términos de $\mathrm{VAB} / \mathrm{PO}$ y de remuneraciones sobre personal ocupado, pero no en términos de remuneraciones sobre personal ocupado o empleo por empresa.

Si bien la mayor parte de los sectores tienen una proporción de I+D sobre ventas menor al promedio, este es el grupo que mayor porcentaje de sectores con un desempeño por encima de la media presenta. Con excepción de informática y electrónica, todos los otros sectores que muestran este comportamiento se corresponden a servicios. La mayor innovación se corresponde al nivel de empresa, en las tres dimensiones, y a producto-mercado.

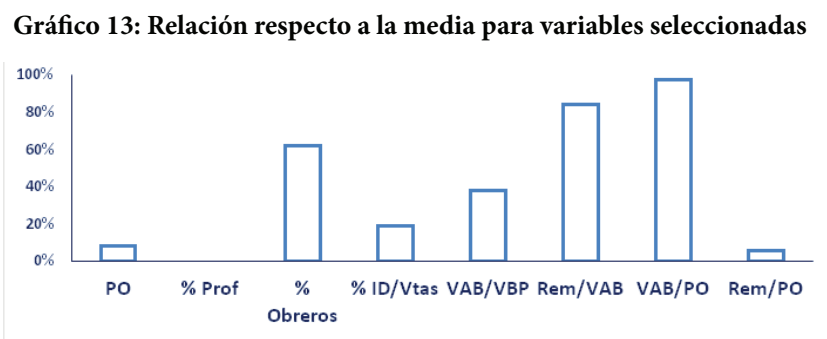

Fuente: Elaboración propia en base a datos INE y ANII

Nota: Se incluyen los sectores para los que se tenía información correspondiente a las variables relevadas con fuente ANII e INE.

\section{Aproximación empírica al impacto de la estructura de los PIP en la estructura productiva}

Dado el interés por evaluar el impacto de la política de PIP sobre el patrón productivo, desde la perspectiva de si contribuye a orientar la economía hacia un estadio de mayor diversificación y complejidad productiva, se realiza una primera exploración de relaciones entre la estructura de los PIP y las variables que, según surge del marco conceptual, capturan efectos de un patrón de desarrollo deseado.

Tal como se refería previamente, en esta primera etapa se analizan además los patrones de innovación de las empresas agrupadas por sector CIIU a 2 dígitos, de modo a identificar si el patrón de inversión de alguna manera se asocia con alguno de los perfiles de innovación identificados.

\subsection{Análisis de correlaciones de PIP con variables clave}

Se estudiaron -como primera aproximación- correlaciones a nivel de variables sectoriales. Dichas variables incluyen en primer lugar las propias de la inversión, y cuatro categorías adicionales relacionadas con características relevantes de una economía diversificada, compleja y dinámica: la innovación, el empleo de calidad, la generación de capacidades empresariales, y la inserción externa.

Los resultados muestran que la estructura de la inversión promovida en 2010-2014 está fuertemente correlacionada con la estructura de activos vigente en el punto de partida (promedio 2008-2010). En este sentido, no se estaría procesando en principio un cambio en la estructura del capital instalado por sectores.

Resulta interesante que esta estructura de inversión promovida está asociada positivamente a sectores con mayor presencia de empresas con capital extranjero, con empresas de mayor tamaño (promedio de ventas), con mayor productividad (tomando como indicador el valor agregado bruto ajustado por impuestos netos de subsidios por persona ocupada), y que pagan mayores remuneraciones por persona ocupada ( $y$ tienen una mayor participación de remuneraciones en el valor agregado como promedio sectorial). Ello parece indicar que las empresas de sectores con estas características son las que hacen (en promedio) mejor uso de los mecanismos de promoción. A su vez, podría asumirse en principio que estas inversiones aportan al dinamismo de la economía y a empleos con mayor remuneración, aunque no necesariamente a sectores que en conjunto sean más innovadores o promuevan la diversificación productiva.

Llamativamente, se encuentra una asociación negativa con el porcentaje de utilización de capacidad instalada en el sector, lo que puede estar indicando a este nivel, que sectores que han perdido capacidad de competencia o participación de mercado cuentan con empresas que están buscando reposicionarse. 
Tabla 2- Síntesis de resultados de correlaciones

\begin{tabular}{|c|c|c|}
\hline & Asociación positiva & Asociación negativa \\
\hline Inversión & $\begin{array}{l}\text { Estructura de activos fijos previa } \\
\text { Volumen de ventas (tamaño) } \\
\text { Presencia de empresas con capital extranjero } \\
\text { VAB por persona ocupada (indicador de productividad) } \\
\text { Remuneraciones promedio (en relación al VAB y por ocupado) }\end{array}$ & Porcentaje uso capacidad instalada \\
\hline Inserción externa & $\begin{array}{l}\text { Innovación (exportaciones con inversión para innovación y para el mundo) } \\
\text { Valor agregado por persona ocupada (productividad) } \\
\text { Remuneraciones promedio (en relación al VAB y por ocupado) }\end{array}$ & $\begin{array}{l}\text { Porcentaje uso capacidad instalada } \\
\text { Exportaciones VAB/VBP }\end{array}$ \\
\hline $\begin{array}{c}\text { Inversión en } \\
\text { capacidades } \\
(1+D+\text { Capacitación })\end{array}$ & $\begin{array}{l}\text { Porcentaje de profesionales y técnicos en personal ocupado } \\
\text { Innovación } \\
\text { VAB/VBP } \\
\text { Intensidad de mano de obra (REM/D) }\end{array}$ & Porcentaje de obreros en personal ocupado \\
\hline Empleo & $\begin{array}{l}\text { Estructura de activos fijos previa } \\
\text { Inversión en bs de capital para innovación (espec. los de alto VAB/PO y Rem } \\
\text { Remuneraciones por ocupado (los de alto VAB/PO) }\end{array}$ & Intensidad de mano de obra (REM/D) \\
\hline Innovación & $\begin{array}{l}\text { Exortaciones sobre ventas } \\
\text { Innovación de varios tipos/ intensidades } \\
\text { Generación de capacidades (I+D + Capacitación) }\end{array}$ & \\
\hline
\end{tabular}

En la medida que la inversión aportara a la diversificación de la economía y a un tejido empresarial más complejo y flexible, ello debería reflejarse en aspectos como la innovación, el empleo de calidad y la generación de capacidades a nivel empresarial. En tal sentido, el análisis de las correlaciones halladas permite identificar algunos aspectos destacables de la asociación entre estas variables:

- Las variables asociadas a actividades de innovación, en sus diversos tipos e intensidades muestran correlación entre ellas, y también con la intensidad exportadora de los sectores y con la inversión en I+D y capacitación.

- Las variables asociadas al empleo, básicamente la estructura por sector, y las asociadas a empleo de calidad, como valor agregado y remuneración por persona ocupada, muestran una correlación positiva con la estructura de activos previa y con la inversión en bienes de capital para la innovación.

- Las variables asociadas a la generación de nuevas capacidades a nivel de empresa, como la inversión en $\mathrm{I}+\mathrm{D}$ (más conocimiento) y en capacitación (recursos humanos más calificados), están asociados con la estructura de personal ocupado en el sector (positivamente con el porcentaje de profesionales y técnicos, y negativamente con el porcentaje de obreros en el total ocupado). La asociación a nivel de sectores, a su vez, es positiva con la innovación y con la proporción de valor agregado bruto (ajustado por impuestos netos de subsidios).

La presencia de empresas con capital extranjero y, especialmente, la intensidad exportadora son aspectos relevantes tanto en términos de innovación como de productividad y empleo de calidad.

\subsection{Perfiles de innovación de los sectores- Análisis por Componentes Principales (ACP)}

Partiendo de la base de la importancia de la actividad innovadora para generar una estructura productiva, se procedió a identificar perfiles de innovación a nivel sectorial (CIIU4 a dos dígitos), para luego evaluar en qué medida la inversión promovida estaba asociada a dichos patrones. La base de datos utilizada fue la encuesta de ANII sobre innovación para 2010-2012, y se utilizaron 12 indicadores que muestran la proporción de empresas que tiene actividades de innovación por CIIU a dos dígitos, combinando 4 tipos y 3 niveles de innovación. La tabla 3 indica qué porcentaje de empresas innova según esos criterios:

Tabla 3: Porcentajes de empresas que innovan según criterios de tipo y nivel de innovación

\begin{tabular}{|l|c|c|c|}
\hline & \multicolumn{3}{|c|}{ Nivel de la innovación } \\
\hline Tipo de innovación & Empresa & Mercado & Internacional \\
\hline Producto & 9,3 & 7,9 & 1,1 \\
\hline Proceso & 16,3 & 5,6 & 0,2 \\
\hline Organización & 12 & 1 & 0,1 \\
\hline Comercialización & 4,5 & 1,7 & 0,1 \\
\hline
\end{tabular}

Fuente: elaborado con datos de la encuesta ANII para industria y servicios 2010-2012

Se aplicó un Análisis en Componentes Principales (ACP) para poner en evidencia la existencia de perfiles de innovación en los diferentes sectores. Se incluyeron en el análisis sectores con más de dos empresas encuestadas e indicadores con valores positivos en más de un sector. A partir de la prueba de KMO y de la de esfericidad de Bartlett 
se concluye que los resultados pueden utilizarse con un enfoque descriptivo. El ACP practicado obtuvo un valor de 0,585 en la prueba de KMO y un sig de 0,00 para la prueba de Bartlett, con comunalidades del orden de 0,6 y más, y una varianza recuperada en las dimensiones significativas de 70,4\%, lo que permite utilizar los resultados con un enfoque descriptivo.

Se identifican cuatro componentes principales que explican el $70 \%$ de la varianza (con autovalor mayor a 1 y quiebre en la pendiente de los autovalores), cuyas características se presentan en las Tablas 4 y 5. En la Tabla 6 se presenta la síntesis de resultados interpretando los cuatro componentes o dimensiones principales y asociándolos a sectores y variables. De acuerdo a los resultados obtenidos, parecería que los niveles de innovación discriminan más los sectores que el tipo de innovación, que recién aparece en el componente 4 .

Tabla 4: Matriz de componentes antes de rotación

\begin{tabular}{|c|ccc|}
\hline \multirow{2}{*}{ Componente } & \multicolumn{3}{|c|}{ Autovalores iniciales } \\
\cline { 2 - 4 } & Total & \% de la varianza & \% acumulado \\
\hline 1 & 2,957 & 29,57 & 29,57 \\
2 & 1,505 & 15,05 & 44,63 \\
3 & 1,333 & 13,33 & 57,96 \\
4 & 1,248 & 12,48 & 70,44 \\
\hline
\end{tabular}

Método de extracción: Análisis de Componentes principales.
Tabla 5: Matriz de componentes antes de rotación

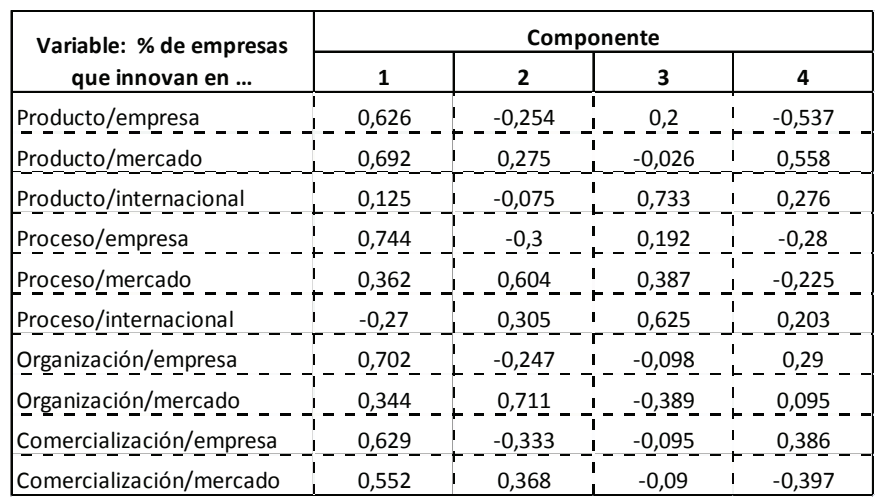

Método de extracción: Análisis de Componentes principales.

En todo caso, el resultado del análisis permite concluir que se identifican a partir de las variables de innovación cuatro componentes principales que corresponden a patrones asociados a las características de la innovación y por tanto a ciertas características sectoriales. Sin embargo no se encuentra una correlación significativa entre dichos patrones de innovación identificados y la inversión promovida a través de la Ley de Promoción de Inversiones y el Régimen de Zonas Francas entre 2010-2014. Ello puede interpretarse como que la inversión privada promovida en dicho período no estuvo asociada a ninguno de los patrones de innovación identificados.

Tabla 6: Síntesis de resultados

\begin{tabular}{|c|c|c|c|c|}
\hline & Componente 1 & Componente 2 & Componente 3 & Componente 4 \\
\hline Varianza explicada & $30 \%$ & $15 \%$ & $13 \%$ & $12 \%$ \\
\hline Caracterización & $\begin{array}{l}\text { Expresa la intensidad de la inno- } \\
\text { vación. Recoge las asociaciones } \\
\text { positivas entre los sectores in- } \\
\text { dicando que tienden a ser más } \\
\text { o menos innovadores en su con- } \\
\text { junto y en el conjunto de los ti- } \\
\text { pos y niveles de innovación, bien } \\
\text { que la innovación internacional } \\
\text { en procesos es la única que se } \\
\text { posiciona negativamente. }\end{array}$ & $\begin{array}{l}\text { Opone innovaciones de nivel de } \\
\text { mercado (dirección positiva) a la } \\
\text { innovación a nivel de empresa, in- } \\
\text { dicando que algunos sectores tien- } \\
\text { den a innovar más en términos de } \\
\text { nivel, cualquiera sea el tipo. }\end{array}$ & $\begin{array}{l}\text { Expresa la intensidad de la inno- } \\
\text { vación, estando en la dirección } \\
\text { positiva los dos tipos de inno- } \\
\text { vación internacional y en nega- } \\
\text { tivo el resto: mercado y empresa, } \\
\text { salvo proceso a mercado }\end{array}$ & $\begin{array}{l}\text { Este componente expresa la } \\
\text { oposición entre los sectores que } \\
\text { tienden a innovar en producto/ } \\
\text { mercado y comercialización/ } \\
\text { empresa versus producto/ } \\
\text { empresa y comercialización/ } \\
\text { mercado pudiendo indicar dos } \\
\text { modalidades de una situación } \\
\text { de desfasaje: un producto nuevo } \\
\text { a nivel de la empresa puede ser } \\
\text { acompanado de una innovación } \\
\text { de comercialización a nivel de } \\
\text { mercado para abordar la compe- } \\
\text { tencia y un producto nuevo para } \\
\text { el mercado puede requerir una } \\
\text { innovación de comercialización } \\
\text { para la empresa. }\end{array}$ \\
\hline $\begin{array}{l}\text { Principales sectores } \\
\text { asociados positiva- } \\
\text { mente }\end{array}$ & $\begin{array}{l}\text { Películas, TV y música; Arqui- } \\
\text { tectura e ingeniería; Telecomuni- } \\
\text { caciones; I+D; Tecnología de in- } \\
\text { formación; Máquinas y equipos; } \\
\text { Caucho y plásticos; Farmacéuti- } \\
\text { cos; Impresión y grabaciones. }\end{array}$ & $\begin{array}{l}\text { Arquitectura e ingeniería; Cueros } \\
\text { y sus productos. }\end{array}$ & $\begin{array}{l}\text { Cueros y sus productos; Far- } \\
\text { macéuticos; Textiles; Informática } \\
\text { y electrónica; Equipo eléctrico. }\end{array}$ & $\begin{array}{l}\text { Máquinas y equipos; Cueros } \\
\text { y sus productos; Tecnología } \\
\text { de información; Telecomuni- } \\
\text { caciones }\end{array}$ \\
\hline $\begin{array}{l}\text { Correlación con } \\
\text { variables de IP }\end{array}$ & No significativa & No significativa & No significativa & No significativa \\
\hline $\begin{array}{l}\text { Correlación (signif- } \\
\text { icativa al } 5 \% \text { ) con } \\
\text { otras variables secto- } \\
\text { riales relevantes }\end{array}$ & $\begin{array}{l}\text { Proporcion de profesionales y } \\
\text { técnicos en personal }(+) \\
\text { Capacitación }(+) \text { e } \mathrm{I}+\mathrm{D} / \text { ventas } \\
(+)\end{array}$ & & $\begin{array}{l}\text { Proporción obreros en personal } \\
(+) \\
\text { Exportaciones/ ventas }(+)\end{array}$ & Monto I+D/ventas (-) \\
\hline
\end{tabular}




\section{El patrón productivo que se perfila a partir de los PIP y su impacto en el desarrollo}

En los últimos diez años en Uruguay se ha registrado un crecimiento excepcional de la inversión, que está asociado en parte a las políticas de promoción. Este trabajo pretende aportar una primera aproximación sectorial al impacto de la inversión promovida en variables clave de la estructura productiva nacional. Desde una perspectiva que asocia un patrón de desarrollo sostenible e inclusivo con la mayor diversificación y complejidad de la matriz productiva, dados sus efectos, entre otros, en el esfuerzo de innovación y en el mercado de trabajo, se entiende que una política de desarrollo pasa por promover una inversión orientada a esos fines. En este marco, la pregunta planteada es si la inversión promovida en Uruguay -entre 2010 y 2014, representativa de un elevado porcentaje de la inversión privada ejecutada, estaría impactando en el patrón de innovación y en indicadores relevantes del mercado de trabajo.

Desde hace décadas Uruguay ha contado con políticas de promoción de la inversión, y sucesivos gobiernos han perfeccionado los instrumentos y han ampliado su aplicación, tanto en materia de actividades productivas como de tipo de empresas elegibles. Esta promoción se enmarca en un sistema de política industrial activa que fue creando una serie de instrumentos focalizados en determinados objetivos y sectores, pero que contribuyen al mismo fin de desarrollo, e indirectamente estimulan la inversión.

Diversos estudios que han analizado la aplicación de esta política concluyen que la misma habría sido efectiva para el logro de sus objetivos primarios o directos. Concluyen que el régimen de promoción se presenta como una herramienta mejorable pero generalmente apropiada para estimular la acumulación de activos fijos en Uruguay, aunque quedan aspectos para mejorar. En este sentido se plantea cuál es el mejor uso de incentivos tributarios, considerando la presencia de externalidades, la eventual atención a fallas de coordinación o en general de mercado, la búsqueda de eficiencia y ciertas formas de equidad, o la priorización de objetivos -por ejemplo recursos humanos vs infraestructura (Llambí,\& Cazulo, 2014).

Uno de los aspectos que no ha sido investigado suficientemente es el impacto sobre la estructura productiva de la aplicación de los instrumentos de política vigentes. El presente trabajo es un primer paso en esa dirección. Cuenta con la limitación de basarse en el nivel sectorial, cuando muchas decisiones se toman a nivel empresarial, y a centrarse en algunas variables. En tal sentido sus resultados son preliminares. Una línea de trabajo futuro es ampliar el análisis al nivel de empresas e incorporar nuevas variables significativas para un patrón de desarrollo sostenible e inclusivo. El trabajo aporta las bases de datos y permite plantear la hipótesis para futuras exploraciones en esta dirección.

Los hechos más novedosos en materia de inversión en los últimos años están asociados a los grandes proyectos de producción de celulosa y a la producción de energía -principalmente eólica. En lo restante, la estructura de inversiones promovidas no parece haber tenido un impacto significativo en términos de cambio de la estructura productiva. La política de promoción asociada a las grandes inversiones sectoriales, al menos en el caso de la energía, ha intentado tener impacto más allá de la inversión en sí, a través de la exigencia de un porcentaje mínimo de integración nacional y de la eventual ganancia de eficiencia asociada al cambio en la matriz energética. En el caso de las plantas de celulosa, el impacto que podría estar asociado al desarrollo de proveedores, no parece haberse producido dado el alto grado de concentración en las compras, incluso a empresas vinculadas a la propia pastera.

Excluyendo energía y celulosa, los diez sectores con un monto de inversión promovida superior al promedio en el período, acumulan el $45 \%$ de la inversión y el 59\% de los proyectos. Los más de cincuenta sectores restantes, acumulan el $17 \%$ del total de inversión promovida en el período y un $37 \%$ de la cantidad de proyectos, dando cuenta de la presencia de proyectos de menor tamaño, más orientados al sector servicios. No parece encontrarse en estos casos un patrón definido, que marque un cambio hacia mayor diversificación y capacidades diferentes.

En el caso específico de innovación, variable clave en un proceso de diversificación y cambio, se identifican cuatro componentes principales que corresponden a patrones asociados a las características de la innovación y por tanto a ciertas características sectoriales. Sin embargo no se encuentra una correlación significativa entre dichos patrones de innovación identificados y la inversión promovida a través de la Ley de Promoción de Inversiones y el Régimen de Zonas Francas entre 2010-2014. Ello puede interpretarse como que la inversión privada promovida en dicho período no estuvo asociada a ninguno de los perfiles de innovación identificados (probablemente porque no estuvo dentro de sus objetivos). Tampoco se encuentra una asociación positiva de los componentes con variables que hacen al mercado laboral, tales como contribución a los puestos de trabajo, o la calidad de empleo (medida por el salario promedio).

Como primera aproximación, puede afirmarse que la inversión promovida está asociada positivamente a sectores con mayor presencia de empresas con capital extranjero, con empresas de mayor tamaño en ventas, con mayor productividad, y que pagan mayores remuneraciones por persona ocupada. Queda pendiente para futuras profundizaciones del estudio en esta línea de análisis, si estas observaciones identificadas nivel de sector se mantendrían en un análisis con datos a nivel de empresas.

Por tanto, como reflexión final queda una primera constatación empírica, que debe ser profundizada, de que la inversión promovida no ha impactado en el patrón productivo sectorial uruguayo introduciendo un sesgo hacia los más innovadores o que generan más y mejores puestos de trabajo. En tal sentido, la política de promoción de inversiones -a pesar de su impacto positivo- no estaría aportando un efecto de diversificación de la estructura productiva orientada hacia un modelo de desarrollo más sostenible e inclusivo. 


\section{Agradecimientos}

Se agradece al Ec. Diego Aguiar, Asistente de investigación del Departamento de Economía, por su contribución a la construcción de la base de datos y apoyo en la edición del artículo.

\section{Referencias}

Agencia Nacional de Investigación e Innovación (2014) Indicadores de Ciencia Tecnología e Innovación en Uruguay. Montevideo: Unidad de Evaluación y Monitoreo, ANII.

Durán, C., Jung, A. \& García, S. (2012). Igualdad de oportunidades y construcción de capacidades para el desarrollo. El caso de Uruguay en O. Jacob (Eds.), Pobreza, Desigualdad de Oportunidades y Politicas Públicas en América Latina (pp. 147-158) Rio de Janeiro: SOPLA Konrad Adenauer Stiftung. (ISBN 9788575041703).

Garbarino, P., Jung, A. y Plottier, P. (2008). La región desde una lógica de clusters: Aportes para construcciones estratégicas en Uruguay. Montevideo: Asociación Cristiana de Dirigentes de Empresa (ACDE) y Fundación Konrad Adenauer.

Gervaz, I. (2012). Impacto de las recientes políticas de promoción de inversiones en Uruguay. Montevideo: Centro de Estudios Fiscales del Uruguay.

Hausmann, R., Hwang, J. \& Rodrik, D. (2007). What you export matters. Journal of Economic Growth, 12(1) 1-25.

Hausmann, R., y Rodrik, D. (2003). Economic development as self discovery. Journal of Development Economics, 72(2) 603-633.

Imbs, J., and Wacziarg, R. (2003). Stages of Diversification. American Economic Review, 93(1), 63-86.

Jung A. \& Vázquez, S. (2013). Desempeño industrial: una reflexión en clave de sectores y empresas exportadoras. En Enfoques sobre estructura productiva, inserción externa y competitividad (pp. 5-82). Montevideo: Konrad Adenauer Stiftung/ ACDE.
Carbajal, F. Carrasco, P. Cazulo, P. Llambí, C. y A. Rius (2014). Una evaluación económica de los incentivos fiscales a la inversión en Uruguay. (Serie Documentos de Trabajo DT No. 20/2014). Montevideo: Instituto de Economía, Facultad de Ciencias Económicas y Administración, Universidad de la República.

Llambí, C. \& Cazulo, P. (2014). Una evaluación económica de los incentivos fiscales a la inversión en Uruguay 2005 - 2012. Montevideo: Centro de Investigaciones Económicas (CINVE), Centro de Estudios Fiscales (CEF).

Peláez, F. (2012). Costo-beneficio de los incentivos tributarios en Uruguay. Montevideo: Centro de Estudios Fiscales del Uruguay.

Plottier, C., Zuasnabar, A. y Castagna, S. (2013). Mapeo de empresas exportadoras de bienes en Uruguay. (Informe de Proyecto). Montevideo: Departamento de Economía, Universidad Católica del Uruguay.

Unidad de Apoyo al Sector Privado - UNASEP. (2014). Cumplimiento de los proyectos promovidos por la Ley 16.906 y sus decretos reglamentarios. Montevideo: Ministerio de Economía y Finanzas.

Vaillant, M. \& Lalanne, A. (2014). Un caso de transformación productiva y comercial, Zonas Francas en Uruguay. Montevideo: CEPAL.

Vázquez, S., Plottier, C. y Zuasnabar, A. (2011). Diversificación y dinamismo exportador ¿Todas son buenas noticias?. En Un puente entre el sector exportador y la academia. (pp. 39-66). Montevideo: Unión de Exportadores del Uruguay y BROU.

Vázquez S. y Jung, A. (2014). Invirtiendo en Uruguay: efectos sobre la matriz productiva de la industria manufacturera. Montevideo: Departamento de Economía, Universidad Católica del Uruguay.

Vázquez, S. (2014). Contexto macroeconómico de la competitividad uruguaya en los últimos 10 años: desempeño y (des)equilibrios. (Documento de Trabajo No. 01/2014). Montevideo: Departamento de Economía, Universidad Católica del Uruguay. 